\title{
Fusobacterium necrogenes
}

National Cancer Institute

\section{Source}

National Cancer Institute. Fusobacterium necrogenes. NCI Thesaurus. Code C86400.

A species of anaerobic, Gram-negative, rod shaped bacteria in the phylum Fusobacteria.

This species is non-spore forming, indole and glutamine decarboxylase negative,

hydrolyzes esculin, and does not reduce nitrate. F. necrogenes is commensal in the human intestinal tract, but is rarely associated with human infections. 